\title{
Diffusion of chiral janus particles in convection rolls
}

\author{
Yunyun $\mathrm{Li},{ }^{1}$ Lihua Li, ${ }^{1}$ Fabio Marchesoni, ${ }^{1,2}$ Debajyoti Debnath, ${ }^{3}$ and Pulak K. Ghosh $\oplus^{3, *}$ \\ ${ }^{1}$ Center for Phononics and Thermal Energy Science, Shanghai Key Laboratory of Special Artificial Microstructure Materials and Technology, \\ School of Physics Science and Engineering, Tongji University, Shanghai 200092, China \\ ${ }^{2}$ Dipartimento di Fisica, Università di Camerino, I-62032 Camerino, Italy \\ ${ }^{3}$ Department of Chemistry, Presidency University, Kolkata 700073, India
}

(Received 7 November 2019; accepted 13 February 2020; published 3 March 2020)

\begin{abstract}
The diffusion of an artificial active particle in a two-dimensional periodic pattern of stationary convection cells is investigated by means of extensive numerical simulations. In the limit of large Péclet numbers, i.e., for self-propulsion speeds below a certain depinning threshold and weak rototranslational fluctuations, the particle undergoes asymptotic normal diffusion with diffusion constant proportional to the square root of its diffusion constant at zero flow. Chirality effects in the propulsion mechanism, modeled here by a tunable applied torque, favors particle jumping between adjacent convection rolls. Roll jumping is signaled by an excess diffusion peak, which appears to separate two distinct active diffusion regimes for low and high chirality. A qualitative interpretation of our simulation results is proposed as a first step toward a fully analytical study of this phenomenon.
\end{abstract}

DOI: 10.1103/PhysRevResearch.2.013250

\section{INTRODUCTION}

Microswimmers are Brownian particles capable of selfpropulsion [1,2]. The simplest category among them consists of artificial micro- and nanopropellers, which, due to some ad hoc asymmetry of their geometry and/or chemical composition, are capable of harvesting environmental energy and convert it into kinetic energy. The artificial microswimmers most investigated in the literature are the so-called Janus particles (JP), basically spherical colloidal particles with two differently coated hemispheres or "faces." Their axial propulsion is sustained by the dipolar near-flow-field they generate by interacting with the surrounding active (mostly highly viscous) medium $[3,4]$.

Recently, artificial microswimmers found promising applications in the pharmaceutical (e.g., smart drug delivery [5]) and medical research (e.g., robotic microsurgery [6]), whereby one expects that the function they are designed to perform is governed in time and space by their diffusive properties. To this regard it is important to control the diffusion of active particles in crowded [4] and patterned environments [7], where they interact with other system components, either chemically [8] or mechanically [9]. Even more important for applications to cell biology and chemical industry is regulating their diffusion in hydrodynamically active mediums $[3,10]$.

To this purpose, we investigated the diffusion of a single overdamped JP with self-propulsion speed $v_{0}$ suspended in a

\footnotetext{
*Corresponding author: gpulakchem@gmail.com

Published by the American Physical Society under the terms of the Creative Commons Attribution 4.0 International license. Further distribution of this work must maintain attribution to the author(s) and the published article's title, journal citation, and DOI.
}

two-dimensional (2D) stationary laminar flow with periodic center-symmetric stream function

$$
\psi(x, y)=\left(U_{0} L / 2 \pi\right) \sin (2 \pi x / L) \sin (2 \pi y / L),
$$

where $U_{0}$ is the maximum advection speed and $L$ the wavelength of the flow unit cell. As illustrated in Fig. 1, $\psi(x, y)$ defines four counter-rotating advection subcells or, following the notation of Ref. [11], convection rolls. Particle transport in such a flow pattern has been studied under diverse conditions. For instance, in the presence of periodic perturbations the deterministic dynamics of a passive particle exhibits remarkable chaotic properties $[12,13]$. Especially relevant to the present work are the results reported for the diffusivity [11] and the nonlinear mobility $[14,15]$ of passive tracers subject to thermal fluctuations. Note that in Ref. [15] the drive acting on the tracer plays the role of a self-propulsion velocity with fixed orientation. However, despite its practical implications, the problem of how a flow field with stream function like $\psi(x, y)$ can affect the diffusion of self-propelled particles has not been fully investigated, yet. The problem was addressed, indeed, by the authors of Ref. [16], but only in the noiseless, chaotic limit. These authors also proved that, for self-propulsion speeds below a certain threshold, the particle gets dynamically trapped inside the convective rolls and its diffusion suppressed.

In this paper, we consider the more realistic situation of an active particle subject to both translational and orientational fluctuations. As a consequence, the direction of its self-propulsion velocity is driven not only by the local flow shear [16] and, possibly, a chiral (applied or intrinsic) torque [17], but also by an intrinsic rotational noise. Moreover, due to thermal fluctuations, random hopping between convection rolls [11] can occur even for self-propulsion speeds below the trapping threshold of Ref. [16]. As a result, active diffusion in the laminar flow of Eq. (1) develops two distinct regimes, 

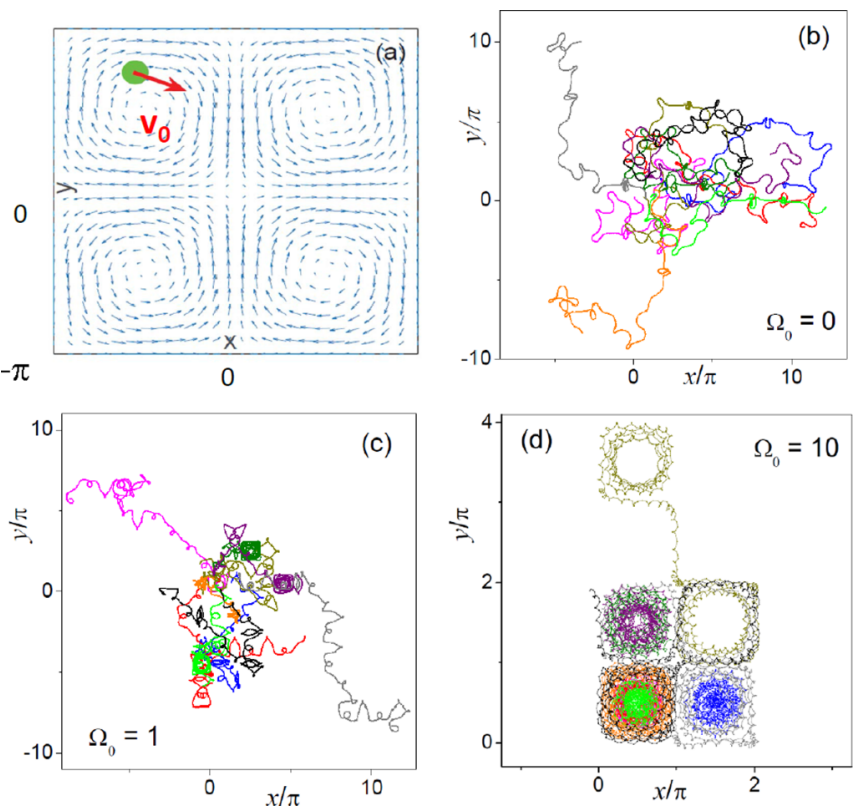

FIG. 1. Diffusion of an active JP in a 2D periodic pattern of convective stationary rolls. (a) Unit flow cell, $\mathbf{v}_{\psi}=\left(\partial_{y},-\partial_{x}\right) \psi$, with $\psi(x, y)$ of Eq. (1), consisting of four counter-rotating subcells. [(b)-(d)] Trajectory samples of length $t=100$, respectively for $\Omega_{0}=$ 0 (achiral), 1 (depolarized), and 10 (highly chiral). Other model parameters are: $\alpha=1, U_{0}=v_{0}=1, D_{0}=D_{\theta}=0.003$, and $L=2 \pi$. Note that for $v_{0}<v_{\text {th }}$, roll jumps are noise activated (see text).

respectively for low and high chirality, both with a peculiar dependence on the particle self-propulsion parameters. At the transition, the chiral and shear torque compensate each other inside two diagonally opposite $\psi(x, y)$ subcells; this causes a partial depinning of the active particle from the convection rolls with a consequent diffusivity surge.

\section{THE MODEL}

In the plane $(x, y)$, the overdamped dynamics of an active JP can be formulated by means of two translational and one rotational Langevin equation (LE)

$$
\begin{aligned}
& \dot{\mathbf{r}}=\mathbf{v}_{\psi}+\mathbf{v}_{0}+\sqrt{D_{0}} \boldsymbol{\xi}(t), \\
& \dot{\theta}=\Omega_{0}+(\alpha / 2) \nabla \times \mathbf{v}_{\psi}+\sqrt{D_{\theta}} \xi_{\theta}(t),
\end{aligned}
$$

where $\mathbf{r}=(x, y),, \mathbf{v}_{\psi}=\left(\partial_{y},-\partial_{x}\right) \psi$ is the advection velocity, and the self-propulsion vector $\mathbf{v}_{0}=v_{0}(\cos \theta, \sin \theta)$ has constant modulus $v_{0}$ and is oriented at an angle $\theta$ with respect to the $x$ axis. The translational noises in the $x$ and $y$ directions, $\boldsymbol{\xi}(t)=\left(\xi_{x}(t), \xi_{y}(t)\right)$, and the rotational noise, $\xi_{\theta}(t)$, are stationary, independent, delta-correlated Gaussian noises, $\left\langle\xi_{i}(t) \xi_{j}(0)\right\rangle=2 \delta_{i j} \delta(t)$ with $i, j=x, y, \theta$. As long as diffusion takes place away from boundaries or other obstacles, the particle can be taken as pointlike. Effects due to its actual geometry and chemical-physical characteristics are encoded in the dynamical parameters appearing in Eq. (2). $D_{0}$ and $D_{\theta}$ are the respective noise strengths, which we assume to be unrelated for generality (e.g., to account for different self-propulsion mechanisms [18]). The reciprocal of $D_{\theta}$ is the correlation, or angular persistence time of $\mathbf{v}_{0}$; accordingly, $v_{0} / D_{\theta}$ quantifies the persistence length of the particle self-propelled random motion. The flow shear exerts a torque on the active particle with frequency proportional to the local fluid vorticity $\nabla \times \mathbf{v}_{\psi}$ [19-21]. The constant $\alpha$ can depend, in principle, on the properties of the particle surface and its fabrication process. Here, for simplicity, we adopt Faxén's second law, which, for a spherical particle, yields $\alpha=1[19,20]$. For $\alpha=1$ and the stream function of Eq. (1), the self-polarization term in the second LE (2) can be conveniently rewritten as $(-1 / 2) \nabla^{2} \psi$ or $(2 \pi / L)^{2} \psi(x, y)$; its modulus is maximum, $\Omega_{l}$, at the center of each subcell. Finally, $\Omega_{0}$ represents a constant torque, either applied by the experimenter [22], or intrinsic to the JP design [23,24], or exerted by a bounding surface [25], or possibly due to unavoidable fabrication defects [6]. In any case, $\Omega_{0}$ is a measure of the particle chirality, which, as proven below, greatly impacts its diffusion. Due to the symmetry of the LE (2) we restrict our analysis to the domain $\Omega_{0} \geqslant 0$.

The LEs (2) can be conveniently reformulated in dimensionless units by rescaling $(x, y) \rightarrow(\tilde{x}, \tilde{y})=(2 \pi / L)(x, y)$ and $t \rightarrow \tilde{t}=\Omega_{L} t$ with $\Omega_{L}=2 \pi U_{0} / L$. Accordingly, the four remaining independent parameters get rescaled as $v_{0} \rightarrow$ $v_{0} / U_{0}, \Omega_{0} \rightarrow \Omega_{0} / \Omega_{L}, D_{0} \rightarrow D_{0} / D_{L}$ and $D_{\theta} \rightarrow D_{\theta} / \Omega_{L}$, with $D_{L}=U_{0} L / 2 \pi$. This means that, without loss of generality, we can set $L=2 \pi$ and $U_{0}=1$ and the simulation results thus obtained can be regarded as expressed in dimensionless units and easily scaled back to arbitrary dimensional units. The stochastic differential Eqs. (2) were numerically integrated by means of a standard Milstein scheme [26]. Particular caution was exerted when computing the asymptotic diffusion constant

$$
D=\lim _{t \rightarrow \infty}\left\langle[x(t)-x(0)]^{2}\right\rangle / 2 t .
$$

Indeed, upon lowering the noise strengths $D_{0}$ and $D_{\theta}$, the intercellular diffusion of a trapped active JP gets suppressed; accordingly, the time transients grow exceedingly long.

We conclude this section with an important remark. With the term particle trapping, we refer to the dynamical trapping caused by advection, which drags the suspended particle along closed orbits. This phenomenon is not to be mistaken with the trapping by an external potential. To this regard, we suggest the reader to compare the problem at hand with the problem of active diffusion in a planar "egg carton" potential [27] or in a square array of truncated harmonic traps [28]. In these two cases, the underlying diffusion process is controlled by thermal activation, whereas in the present problem a crucial role is played by advection.

\section{RESULTS}

In this paper, we focus on the phenomenon of advection dominated active diffusion, that is on the dynamical regime where a noiseless achiral JP would be strictly localized by the stream function $\psi(x, y)$. Indeed, depinning of a noiseless particle from the dynamical trap represented by a single convection roll occurs for self-propulsion speeds above a certain threshold [16]. For a qualitative estimate of such a depinning speed $v_{\text {th }}$, we notice that a trapped active particle can only perform circular orbits with radius $v_{0} / \Omega_{L}$ not exceeding the effective half-width of the convection roll, $R_{\mathrm{S}}$. For a square subcell of $\psi(x, y)$, Eq. (1), $R_{\mathrm{S}} \simeq L / 2 \sqrt{2}$; hence, the trapping condition $v_{0}<v_{\mathrm{th}}=\Omega_{L} R_{\mathrm{S}}$. For the flow parameters used 
here, $v_{\text {th }} \simeq 2.2$, in close agreement with the numerical result obtained in Ref. [16].

\section{A. Convection rolls as dynamical traps}

The laminar flow exerts opposite shear (or selfpolarization) torques in each pair of neighboring $\psi(x, y)$ subcells. Accordingly, we term the convection rolls positive or negative, depending on the sign of the particle selfpolarization. As all simulation data reported here are for $\alpha=1$, vorticity and self-polarization have the same signpositive in the subcells centered at $( \pm L / 2)(1,1)$ and negative in the subcells centered at $( \pm L / 2)(1,-1)$. Of course, the chiral torque, $\Omega_{0}$, in the second Eq. (2) has a different impact on the particle dynamics, depending on the sign of the subcell considered. In a negative subcell, a positive chiral torque with $\Omega_{0} \simeq \Omega_{L}$ tends to annul the self-polarization torque. As a result, the "depolarized" particle can escape the subcell even for $v_{0}<v_{\text {th }}$ and, in the absence of noise, to sojourn inside one of the positive subcells. This mechanism is termed here partial depinning because it is limited to the negative rolls, in contrast with the depinning occurring globally for $v_{0}>$ $v_{\text {th }}$ [16]. This situation is graphically illustrated in Fig. 1 . For $\Omega_{0} \ll \Omega_{L}$, the particle trajectories wander across the square array of convection rolls undergoing marked changes of direction upon crossing them. It is only for $\Omega_{0} \sim \Omega_{L}$, that they start spiraling, but only inside the positive subcells, panel (c). Finally, for $\Omega_{0} \gg \Omega_{L}$, trajectories appear to be the superposition of advection and counterclockwise chiral rotations, the chiral rotations having much shorter a curvature radius than the advection ones, panel (d). Therefore a highly chiral particle is trapped inside the convection rolls most of the time, irrespective of their vorticity sign. As quantitatively confirmed by the numerical data presented below, the characteristic self-polarization frequency, $\Omega_{L}$, thus separates two distinct chirality regimes, respectively, of low, $\Omega_{0} \ll \Omega_{L}$, and high chirality, $\Omega_{0} \gg \Omega_{L}$.

Based on this argument, we expect that the particle stationary probability density (pdf), $P(x, y)$, tends to accumulate inside the positive subcells. To this purpose, we integrated $P(x, y)$ over the positive and negative subcells, separately, obtaining respectively the quantities $P_{+}$and $P_{-}$plotted in Fig. 2. Of course, being all pdf normalized to 1 , in the absence of chiral depinning, $P_{+}=P_{-}=1 / 4$. A strong spatial asymmetry of $P(x, y)$ emerges as $\Omega_{0}$ grows larger than $\Omega_{L}$.

In the noiseless limit, $D_{0}=D_{\theta}=0$, depinning occurs slightly below the self-polarization frequency, that is for $\Omega_{0} \simeq 0.9 \Omega_{L}$. This is consistent with the remark that $\Omega_{L}$ denotes the maximum vorticity at the center of the $\psi(x, y)$ subcells. On the other hand, we also notice that angular fluctuations with finite strength, $D_{\theta}>0$, no matter what their physical origin, weaken the effect of the chiral torque $\Omega_{0}$. Consequently. in the presence of noise, the onset of partial depinning from the negative $\psi(x, y)$ subcells gets "delayed," that is, $P_{ \pm}$deviate substantially from their uniform-distribution value, $1 / 4$, only for larger $\Omega_{0}$ values, i.e., $\Omega_{0}>\Omega_{L}$.

\section{B. Advection controlled diffusion}

The strong chirality dependence of the trajectories shown in Figs. 1(b)-1(d) and their nonuniform spatial localization illustrated in Fig. 2, have an immediate impact on the

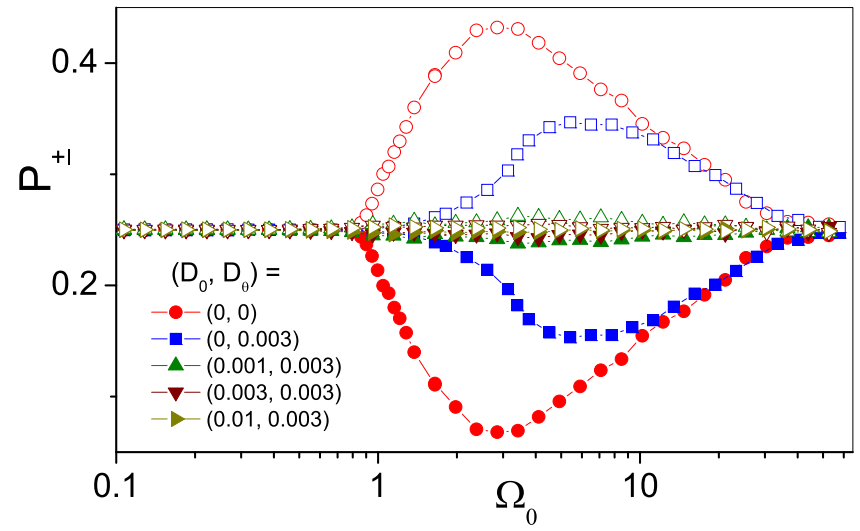

FIG. 2. Particle probability density integrated respectively over a positive $\left(P_{+}\right.$, solid symbols $)$and a negative subcell $\left(P_{-}\right.$, empty symbols), vs $\Omega_{0}$ for different values of the noise strengths, $D_{0}$ and $D_{\theta}$ (see legend). Other model parameters are $\alpha=1, U_{0}=v_{0}=1$, and $L=2 \pi$. Particle depinning from the negative subcells occurs for $\Omega_{0} \sim \Omega_{L}$.

particle asymptotic diffusion constant, $D$. For a particle with $v_{0} / v_{\text {th }} \ll \Omega_{0} / \Omega_{L}$ the persistence length of its trajectories is much shorter than the flow cell size, $v_{0} / \Omega_{0} \ll L / 2 \pi$, so that its intracell diffusion constant is well approximated by

$$
\bar{D}_{\infty}=D_{0}+\frac{v_{0}^{2}}{2 D_{\theta}} \frac{1}{1+\left(\Omega_{0} / D_{\theta}\right)^{2}},
$$

that is the diffusion constant of a chiral active particle in the absence of advection [17,23].

We know [16] that an achiral active particle, $\Omega_{0}=0$, with $v_{0}<v_{\text {th }}$, crosses the subcell separatrices, Fig. 1(b), only as an effect of its roto-translational fluctuations. On keeping ignoring advection, its diffusion constant then would consist again of a translational term, $D_{0}$, due to thermal noise and an additional term from intercell jumps with effective step $L / 2 \pi$, namely, [29]

$$
\bar{D}_{0}=D_{0}+D_{L}+\frac{v_{0}}{2 U_{0}} .
$$

We have now to take into account that diffusion occurs here in a flow pattern of stream function $\psi(x, y)$. The effects of advection on the diffusion of an active JP is illustrated in Fig. 3. To interpret the numerical results displayed there we notice that for $v_{0} \ll U_{0}$ (trapped particle) and $D_{0} \ll D_{L}$ (weak noise-induced depinning, see Sec. III C), both the high- and low-chirality diffusion constants $\bar{D}_{\infty}$, Eq. (3), and $\bar{D}_{0}$ Eq. (4), are much smaller than $D_{L}$ (large effective Péclet number [11]). This suggests that Eq. (38) of Ref. [11] may apply to the case of active particles, too. A simple extension of that equation to the stream function $\psi(x, y)$ of Eq. (1) yields the approximate fitting formula,

$$
D=\left(D_{L} \bar{D}\right)^{1 / 2},
$$

that is, for large Péclet numbers, the advective diffusion constant is proportional to the square root of the no-flow particle diffusion constant, $\bar{D}$. The quantity $\bar{D}$ is approximated by Eqs. (3) and (4), respectively, in the high and low chirality regimes. 


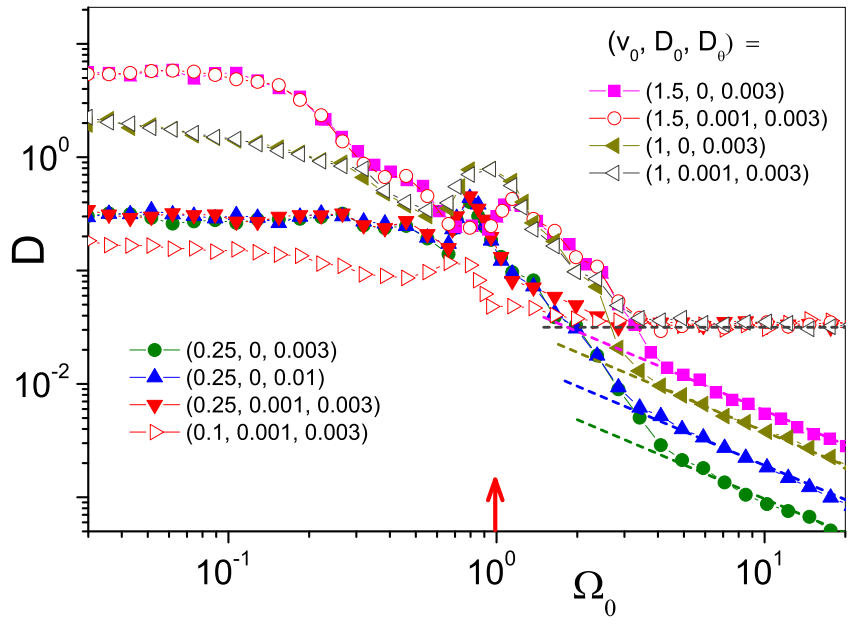

FIG. 3. Diffusion $D$ vs chiral torque $\Omega_{0}$ in the cellular flow of Eq. (1) for different values of $D_{0}, D_{\theta}$, and $v_{0}$ (see legends). Other model parameters are $\alpha=1, U_{0}=1$, and $L=2 \pi$. Dashed horizontal and sloped lines represent our analytical prediction, Eqs. (3)-(5). The vertical arrow corresponds to the condition $\Omega_{0}=\Omega_{L}$.

In Fig. 3, the low and high chirality regimes are separated by an excess diffusion peak centered at around $\Omega_{0} \simeq \Omega_{L}$ (with a weak dependence on $v_{0}$ ). This is the signature [30] of particle depinning from the negative subcells anticipated above. At high $\Omega_{0}$, the data set plotted in Fig. 3 exhibit tails of two kinds, depending on the value of $D_{0}$ : (i) horizontal plateaus, $D=\left(D_{L} D_{0}\right)^{1 / 2}$, insensitive to the self-propulsion parameters, for finite thermal noise, and (ii) $D \simeq\left(D_{\theta} D_{L} / 2\right)^{1 / 2} v_{0} / \Omega_{0}$ for vanishingly translational noise, $D_{0}=0$. Both behaviors are closely reproduced by Eq. (5) after replacing $\bar{D}$ with $\bar{D}_{\infty}$ of Eq. (3) (dashed lines).

The validity of Eq. (5) in the regime of high chirality, $\Omega_{0} \gg \Omega_{L}$, is also apparent in Fig. 4. For $D_{0}=0$, the predicted

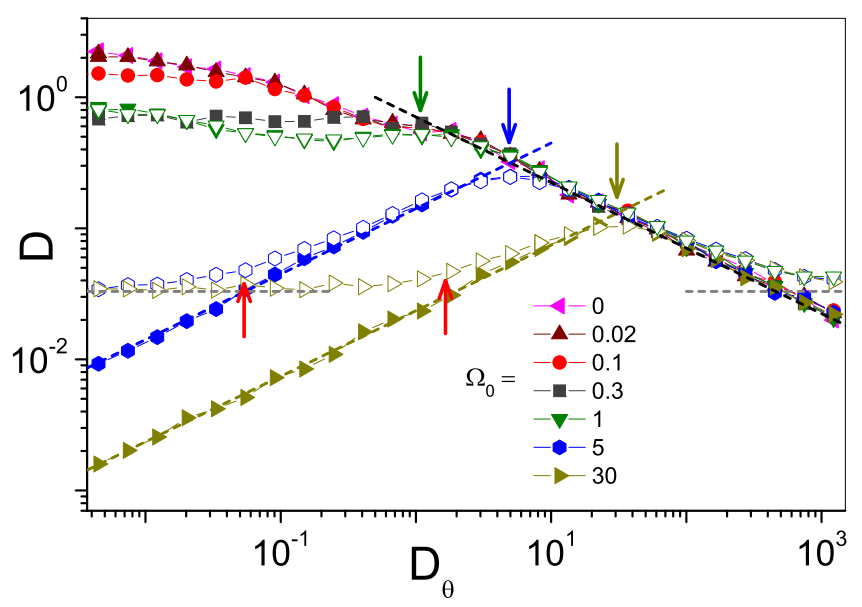

FIG. 4. Diffusion $D$ vs rotational noise strength $D_{\theta}$ in the cellular flow of Eq. (1) for $D_{0}=0$ (solid symbols) and 0.001 (empty symbols) and different $\Omega_{0}$ (see legend). Other model parameters are $\alpha=1, U_{0}=1$, and $L=2 \pi$. Dashed lines represent analytical predictions based on Eqs. (3) and (5). Downward and upward vertical arrows locate respectively the predicted maxima at $D_{\theta}=\Omega_{0}$ and the emergence of the finite $D_{0}$ plateau at low $D_{\theta}$ (see text).

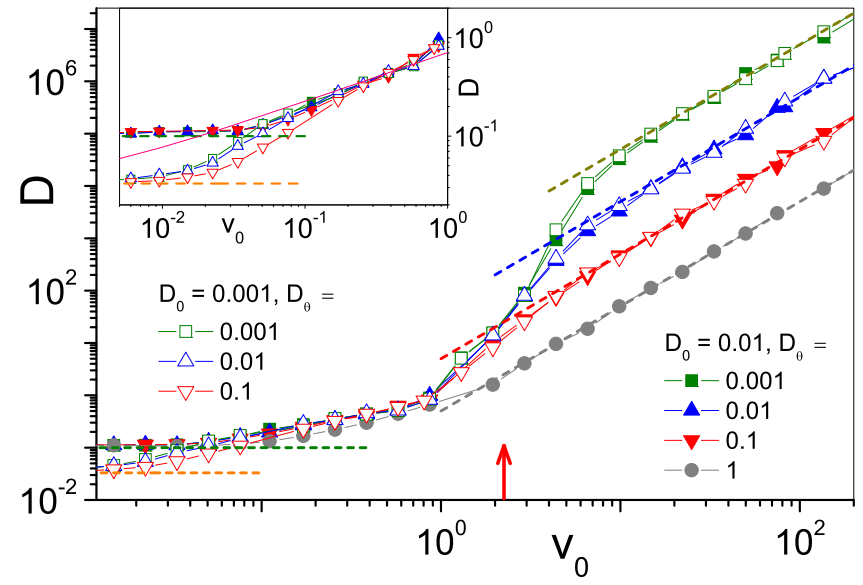

FIG. 5. Diffusion $D$ vs self-propulsion speed $v_{0}$ in the cellular flow of Eq. (1) for $\Omega_{0}=0$ and different $D_{0}$ and $D_{\theta}$; (see legend and inset for more details at low $v_{0}$ ). Other model parameters are $\alpha=1$, $U_{0}=1$, and $L=2 \pi$. Dashed horizontal and sloped lines represent our analytical prediction (see text). The vertical arrow corresponds to the condition $v_{0}=v_{\text {th }}=2.2$ (see text) [16].

diffusion constant $\left(D_{L} \bar{D}_{\infty}\right)^{1 / 2}$, Eqs. (3)-(5), grows like $D_{\theta}^{1 / 2}$ for $D_{\theta}<\Omega_{0}$, and then decays like $D_{\theta}^{-1 / 2}$ for $D_{\theta}>\Omega_{0}$, after going through a maximum at $D_{\theta}=\Omega_{0}$ (downward arrows). Our formula for $D$ fits closely the simulation data over the entire $D_{\theta}$ domain of Fig. 4 . For finite $D_{0}$, both the raising and decaying branches are still visible, except they appear to merge into the thermal plateau with $D=\left(D_{L} D_{0}\right)^{1 / 2}$. This happens when the diffusion term due to self-propulsion grows negligible with respect to $D_{0}$. Accordingly, for instance, at low $D_{\theta}$ the thermal plateau extends up to $D_{\theta} \sim 2\left(\Omega_{0} / v_{0}\right)^{2} D_{0}$ (upward arrows).

In the low chirality regime, $\Omega_{0}<\Omega_{L}$, the raising branch of the $D$ curves of Fig. 4 is replaced by a horizontal branch, which weakly depends on the angular frequencies, $\Omega_{0}$ and $D_{\theta}$. Moreover, the horizontal and the decaying branches of the low chirality $D$ curves connect around $D_{\theta} \sim \Omega_{L}$. Indeed, when the chiral frequency, $\Omega_{0}$, is lowered below the self-polarization frequency, the natural frequency for the angular rate, $D_{\theta}$, to compare with is now $\Omega_{L}$. For $\Omega_{0}>\max \left\{\Omega_{L}, D_{\theta}\right\}$ the JP behaves as a regular Brownian particle with effective local diffusion constant, $D_{0}+v_{0}^{2} / 2 D_{\theta}$, see Eq. (3); the $D$ decaying branch is therefore the same for both low and high chirality.

Such a distinct diffusion regime is better illustrated in Fig. 5, where we study the constant $D$ as a function of the selfpropulsion speed, $v_{0}$. As expected, for $v_{0} \gg v_{\text {th }}$, the particle is largely insensitive to the advective drag, so that its diffusion constant approaches the zero-flow value, $\bar{D}_{\infty}$, of Eq. (3), i.e., $D$ is quadratic in $v_{0}$ (sloped dashed lines). The curves plotted in the main panel of Fig. 5 show a sharp jump in the vicinity of the threshold, $v_{\text {th }}$, thus confirming the existence of the depinning mechanism introduced in the noiseless limit by the authors of Ref. [16]. Relevant to the present study is the $v_{0}$ dependence of $D$ below the depinning threshold, $v_{0}<v_{\text {th }}$. As anticipated above, we expect that formula (5) applies to a flow trapped particle also at low chirality, $\Omega_{0}<\Omega_{L}$, provided that $\bar{D}$ is replaced by $\bar{D}_{0}$ of Eq. (4). For vanishing values of $v_{0}$, we recover the expected limit $\left(D_{0} D_{L}\right)^{1 / 2}$ (horizontal 


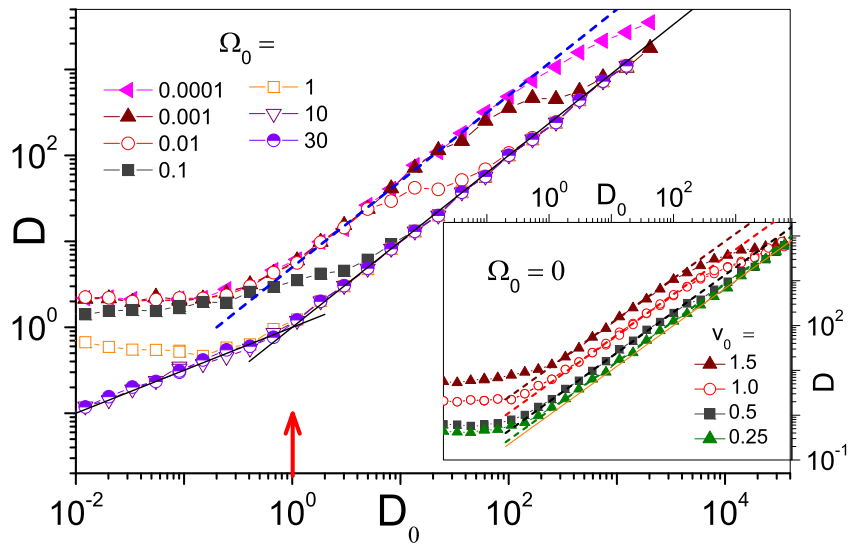

FIG. 6. Diffusion $D$ vs translational noise $D_{0}$ in the cellular flow of Eq. (1) for $D_{\theta}=0$ and main panel: $v_{0}=1$ and different $\Omega_{0}$, inset: $\Omega_{0}=0$ and different $v_{0}$ (see legends). Other model parameters are: $\alpha=1, U_{0}=1$, and $L=2 \pi$. Straight lines represent the analytical predictions discussed in the text for high (solid) and low chirality (dashed). A vertical arrow locates $D_{0}=D_{L}$.

dashed lines), whereas for $D_{0} / D_{L} \ll v_{0} / U_{0} \ll 1$ the constant $D$ grows proportional to $v_{0}^{1 / 2}$ (inset of Fig. 5). We remind here that in the noiseless limit, $D_{0}, D_{\theta} \rightarrow 0$, the particle dynamics becomes chaotic [16] and diffusion is suppressed (and hard to compute numerically).

\section{Noise controlled diffusion}

The effects discussed in the foregoing subsections are detectable only at low thermal noise levels. In Fig. 6, we illustrate how advection effects can be washed out by large thermal noise, even under the depinning threshold, i.e., for $v_{0}<v_{\text {th }}$. In the case of a high chirality particle, $\Omega_{0} \gg \Omega_{L}$, at low noise, $D_{0} \ll D_{L}$, we know that $D=\left(D_{L} D_{0}\right)^{1 / 2}$, whereas for exceedingly large $D_{0}$, we expect $D=D_{0}$ (free diffusion limit). The transition between these two limits would take place for $D_{0} \sim D_{L}$. This is consistent with the simulation data of Fig. 6 (vertical arrow). The case of low chirality is more interesting. The large noise branch of $D$ till sets out proportional to $D_{0}$, but with substantially larger slope, which seems to increase proportionally to $v_{0}^{2}$ (see inset). Our numerical data for $D$ finally approach the free diffusion law, $D=D_{0}$, but asymptotically, only, around $D_{0} \sim U_{0}^{2} / \Omega_{0}$. To explain this phe- nomenon we remark that at low Péclet numbers, $D_{0} \gg D_{L}$, the active particle is no longer trapped in the convection rolls. For low angular rates, $D_{\theta}, \Omega_{0} \ll \Omega_{L}$, its mean free path is of the order of $v_{0} \tau_{L}$, where $\tau_{L}=L / 2 \pi U_{0}$ is the effective cell crossing time, and gets scattered against the cell separatrix with (short) persistence time, $\tau_{0}=(L / 2 \pi)^{2} / 8 D_{0}$, governed by the translational noise. Using the argument invoked to derive Eq. (4), we predict, $D=D_{0}\left[1+4\left(v_{0} / U_{0}\right)^{2}\right]$, in good agreement with the simulation data reported in Fig. 6. By the same token, one locates the switching between such a transient law and the free diffusion law at around $D_{0} \sim U_{0}^{2} / 8 \Omega_{0}$.

In conclusion, our simulations prove that the combination of advection and self-propulsion determines an appreciable excess diffusion of weakly chiral active JPs even at low Péclet numbers.

\section{CONCLUSIONS}

In this paper, we have shown how active particles in hydrodynamically active mediums exhibit peculiar diffusion properties, which distinguish them from common colloidal particles [31-33]. This is particularly true in the low chirality regime, where self-propulsion determines a rich phenomenology of the diffusion process. We remind that the simple and best known stream function $\psi(x, y)$ of Eq. (2), models situations that have been already implemented experimentally, e.g., with rotating cylinders [13] or with ion solutions in arrays of magnets [34]. Moreover, the numerical and analytical techniques reported here can be easily extended to different stream functions to represent convection rolls of varying topologies [35]. It is clear from this investigation that, in view of technological applications, advection controlled diffusion should be considered as an effective tool to govern the transport of active matter. Important examples are microfluidic devices [36] or even microswimmer diffusion in steady turbulent flows [34].

\section{ACKNOWLEDGMENTS}

Y.L. is supported by the NSF China under Grants No. 11875201 and No. 11935010. P.K.G. is supported by SERB Start-up Research Grant (Young Scientist) No. YSS/2014/000853 and the UGC-BSR Start-Up Grant No. F.30-92/2015. D.D. thanks CSIR, New Delhi, India, for support through a Junior Research Fellowship.
[1] Janus Particle Synthesis, Self-Assembly and Applications, edited by S. Jiang and S. Granick (RSC, Cambridge, 2012).

[2] A. Walther and A. H. E. Müller, Janus particles: Synthesis, selfassembly, physical properties, and applications, Chem. Rev. 113, 5194 (2013).

[3] M. C. Marchetti, J. F. Joanny, S. Ramaswamy, T. B. Liverpool, J. Prost, M. Rao, and R. A. Simha, Hydrodynamics of soft active matter, Rev. Mod. Phys. 85, 1143 (2013).

[4] J. Elgeti, R. G. Winkler, and G. Gompper, Physics of microswimmers, single particle motion and collective behavior: a review, Rep. Prog. Phys. 78, 056601 (2015).
[5] See, e.g., Smart Drug Delivery System, edited by A. D. Sezer (IntechOpen, 2016).

[6] J. Wang, Nanomachines: Fundamentals and Applications (Wiley-VCH, Weinheim, 2013).

[7] G. Volpe, I. Buttinoni, D. Vogt, H.-J. Kümmerer, and C. Bechinger, Microswimmers in patterned environments, Soft Matter 7, 8810 (2011).

[8] T. Debnath, P. K. Ghosh, Y. Li, F. Marchesoni, and F. Nori, Active diffusion limited reactions, J. Chem. Phys. 150, 154902 (2019).

[9] G. Minño, T. E. Mallouk, T. Darnige, M. Hoyos, J. Dauchet, J. Dunstan, R. Soto, Y. Wang, A. Rousselet, and E. Clement, 
Enhanced Diffusion Due to Active Swimmers at a Solid Surface, Phys. Rev. Lett. 106, 048102 (2011).

[10] E. Lauga and T. R. Powers, The hydrodynamics of swimming microorganisms, Rep. Prog. Phys. 72, 096601 (2009).

[11] W. Young, A. Pumir, and Y. Pomeau, Anomalous diffusion of tracer in convection rolls, Phys. Fluids A 1, 462 (1989).

[12] T. H. Solomon and J. P. Gollub, Chaotic particle transport in time-dependent Rayleigh-Bénard convection, Phys. Rev. A 38, 6280 (1988).

[13] T. H. Solomon and I. Mezić, Uniform resonant chaotic mixing in fluid flows, Nature (London) 425, 376 (2003).

[14] Y.-N. Young and M. J. Shelley, Stretch-Coil Transition and Transport of Fibers in Cellular Flows, Phys. Rev. Lett. 99, 058303 (2007); H. Manikantan and D. Saintillan, Subdiffusive transport of fluctuating elastic filaments in cellular flows, Phys. Fluids 25, 073603 (2013).

[15] A. Sarracino, F. Cecconi, A. Puglisi, and A. Vulpiani, Nonlinear Response of Inertial Tracers in Steady Laminar Flows: Differential and Absolute Negative Mobility, Phys. Rev. Lett. 117, 174501 (2016).

[16] C. Torney and Z. Neufeld, Transport and Aggregation of SelfPropelled Particles in Fluid Flows, Phys. Rev. Lett. 99, 078101 (2007)

[17] S. vanTeeffelen and H. Löwen, Dynamics of a Brownian circle swimmer, Phys. Rev. E 78, 020101(R) (2008).

[18] P. K. Ghosh, V. R. Misko, F. Marchesoni, and F. Nori, SelfPropelled Janus Particles in A Ratchet: Numerical Simulations, Phys. Rev. Lett. 110, 268301 (2013).

[19] G. B. Jeffery, The motion of ellipsoidal particles immersed in a viscous fluid, Proc. R. Soc. A 102, 161 (1922).

[20] A. Zöttl and H. Stark, Nonlinear Dynamics of a Microswimmer in Poiseuille Flow, Phys. Rev. Lett. 108, 218104 (2012).

[21] A. Geiseler, P. Hänggi, and F. Marchesoni, Self-polarizing microswimmers in a ctive density waves, Sci. Rep. 7, 41884 (2017).

[22] T. R. Kline, W. F. Paxton, T. E. Mallouk, and A. Sen, Catalytic nanomotors: Remote-controlled autonomous movement of striped metallic nanorods, Angew. Chem., Int. Ed. 44, 744 (2005).
[23] D. Debnath, P. K. Ghosh, Y. Li, F. Marchesoni, and B. Li, Diffusion of eccentric microswimmers, Soft Matt. 12, 2017 (2016).

[24] X. Wang, M. In, Ch. Blanc, A. Würger, M. Nobili, and A. Stocco, Janus colloids actively rotating on the surface of water, Langmuir 33, 13766 (2017).

[25] A. Zöttl and H. Stark, Emergent behavior in active colloids, J. Phys.: Condens. Matter 28, 253001 (2016).

[26] P. E. Kloeden and E. Platen, Numerical Solution of Stochastic Differential Equations (Springer, 1992).

[27] A. Nourhani, V. H. Crespi, and P. E. Lammert, Guiding Chiral Self-Propellers in a Periodic Potential, Phys. Rev. Lett. 115, 118101 (2015).

[28] W. Yang, V. R. Misko, F. Marchesoni, and F. Nori, Colloidal transport through trap arrays controlled by active microswimmers, J. Phys.: Condens. Matter 30, 264004 (2018).

[29] D. ben-Avraham and S. Havlin, Diffusion and Reactions in Fractals and Disordered Systems (Cambridge University Press, Cambridge, 2000).

[30] G. Costantini and F. Marchesoni, Threshold diffusion in a tilted washboard potential, Europhys. Lett. 48, 491 (1999).

[31] B. Wang, S. M. Anthony, S. C. Bae, and S. Granick, Anomalous yet Brownian, Proc. Natl. Acad. Sci. USA 106, 15160 (2009).

[32] M. V. Chubynsky and G. W. Slater, Diffusing Diffusivity: A Model for Anomalous, Yet Brownian, Diffusion, Phys. Rev. Lett. 113, 098302 (2014).

[33] Y. Li, F. Marchesoni, D. Debnath, and P. K. Ghosh, NonGaussian normal diffusion in a fluctuating corrugated channel, Phys. Rev. Res. 1, 033003 (2019).

[34] P. Tabeling, Two-dimensional turbulence: a physicist approach, Phys. Rep. 362, 1 (2002).

[35] A. Crisanti and A. Vulpiani, On the effects of noise and drift on diffusion in fluids, J. Stat. Phys. 70, 197 (1993).

[36] T. M. Squires and S. R. Quake, Microfluidics: Fluid physics at the nanoliter scale, Rev. Mod. Phys. 77, 977 (2005). 